\title{
MÁS ALLÁ DE OCCIDENTE. LO QUE LA ANTROPOLOGÍA PUEDE ENSEÑAR A LA FILOSOFÍA \\ Vanesa Blanco Gallardo*
}

La pretensión de este artículo, presentado en un simposio sobre Teorías de la verdad, es la de acercar unas breves pinceladas sobre lo que la antropología hace y puede decir, a otras disciplinas, en este caso concreto, a la esfera del saber filosófico. El centro de todo el discurso que en adelante voy a realizar será un esfuerzo por dejar claro que el conocimiento no está fuera de la contextualización de un grupo social determinado, y que por tanto, los universales que tantas teorías epistemológicas nos han mostrado a lo largo de la historia de la filosofía no son más que aplicables dentro de un esquema determinado cultural, pero no siempre y en todo lugar exportables o de aplicabilidad universal. Este es el motor interno que pretendo conseguir que se plasme en el interior de la argumentación posterior

\section{Palabras claves:}

Filosofía, antropología, verdad, etnografía, relativismo cultural, relativismo lingüístico, relativismo cognitivo

The aim of this article, presented at a symposium on Theories of Truth, is to bring a few hints about what anthropology does and what can it say to other disciplines, in this case, to the field of philosophical knowledge. The center of the whole discourse will be an effort to make clear that knowledge is not out of the contextualization of a particular social group, and therefore, the universals, which many epistemological theories have shown us over history of philosophy are only applicable within a certain cultural base, but not always and everywhere exportables or universally applicables. This is the engine which I intend to capture within the subsequent argument.

*Universidad Complutense de Madrid. aisovela5@hotmail.com 


\section{Keywords:}

Philosophy, anthropology, truth, ethnography, cultural relativism, linguistic relativism, cognitive relativism

\section{Resumen de contenidos:}

1. Introducción

2. De la filosofía a la antropología de la mano de Nietzsche y Wittgenstein
2.1. Nietzsche

2.2. Wittgenstein

2.3. El tiempo como ejemplo

3. Qué es eso de la antropología

3.1. Precisando el concepto

3.2. Líneas históricas

3.3. Metodología

3.3.1. Técnicas

3.3.2. Mirada antropológica

3.3.3. Análisis

3.4. Consideraciones sobre la cientificidad

4. Relativismo lingüístico, cognitivo y cultural

4.1. Hipótesis Sapir-Whorf

4.2. El "pensamiento salvaje" de Lévi-Strauss

5. Conclusión (o posible punto de partida)

\section{Introducción}

Empezaré por mostrar como este modo de hacer etnográfico, propio de la antropología, que pretendo dar a conocer, y lo determino desde este momento como otra alternativa epistemológica (no la única, ni la mejor), que puede ser sugerida (al menos teóricamente) desde la filosofía a través de dos obras con las que comenzaré el camino, Sobre verdad y mentira en sentido extramoral de Friedrich Nietzsche e Investigaciones filosóficas de Ludwig Wittgenstein.

En lo siguiente, y de forma bastante breve, mostraré de que hablo cuando digo "antropología", así como algunas de las líneas históricas de la disciplina, sin más propósito que el de abrir una nueva ventada de conocimiento a los legos en la materia que pueda permitirles 
el conocimiento sobre otra forma de hacer ciencia e investigar que pueda enseñarles otras formas de conocer. Sí me detendré más en el aspecto metodológico de la etnografía, que es el que puramente incide en el modo en que los antropólogos conocemos: qué técnicas son las propias de la disciplina, qué es lo que conocemos y cómo lo conocemos son algunas de las preguntas a las que dará respuesta este apartado.

Después terminaré en un apartado conclusivo en el que a través de lo expuesto anteriormente, examinaremos como la apuesta que hago en este artículo es por un relativismo cognitivo que reta a las teorías epistemológicas definitivas que se nos muestran en las sucesivas gnoseologías filosóficas occidentales. Intentaré poner de manifiesto como hay otros tipos de realidades cognoscibles que no son las que la historia de la filosofía nos ha transmitido.

\section{De la filosofía a la antropología de la mano de Nietzsche y Wittgenstein}

\subsection{Nietzsche}

En su obra Sobre verdad y mentira Nietzsche sostiene la idea del conocimiento como invención del hombre, invención que a su vez es un recurso para la supervivencia del género humano, en tanto que a través del conocimiento y de la dicotomía verdadero/falso puede llegar a conseguir un orden de realidad soportable para su vida. En el argumento del autor una de las frases que más poéticamente acentúa lo que es la verdad es la siguiente:

“QQué es entonces la verdad? Un hueste en movimiento de metáforas, metonimias y antropomorfismos, en resumidas cuentas, una serie de relaciones humanas que han sido realzadas, extrapoladas y adornadas poética y retóricamente y que, después de un prolongado uso, un PUEBLO considera firmes, canónicas y vinculantes; las verdades son ilusiones de las que se ha olvidado que lo son, metáforas que se han vuelto gastadas y sin fuerza sensible, monedas que han perdido su troquelado, y no son ya consideradas como monedas, sino como metal" (la cursiva es mía)

Más que profundizar en esta idea sobre la verdad, según el autor, lo que más interés despierta en mí, y sobre el que quiero llamar la atención, es sobre el aspecto reseñado en cursiva en el fragmento. Parece

${ }^{1}$ Nietzsche, Sobre verdad y mentira en sentido extramoral, Tecnos, Madrid, 2007, p 25 
quedar bastante claro que el uso que se nos muestra de la verdad en tanto "aquello que es susceptible de ser conocido" es aquello que un pueblo considera como tal. Por lo tanto lo que es verdad para cualquiera de los españoles, quizá no sea tan cierto para los bororo. Debido a que el uso de una determinada verdad se transmite a lo largo de generaciones, ¿cómo sentenciar que lo que estos bororo consideran verdadero no es tan cierto como nosotros consideramos como tal?. ¿Qué o quién dicta lo que es verdad? ¿Y desde qué lugar geográfico o posición lo hace? Creo que estas preguntas son bastantes ilustrativas del cuestionamiento que pretendo hacer acerca de las diversas teorías de la verdad absolutas ${ }^{2}$. Propongo que de aquí en adelante, sino son demostrables, en el sentido de universalizables, entendamos las distintas teoría de la verdad como teorías parciales, o restringidas a un determinado grupo socio cultural.

En otro momento de esta pequeña gran obra de Nietzsche podemos encontrar una referencia a otro modo de conocimiento, de percepción, leamos al maestro:

“[...] gracias solamente al hecho de que el hombre se olvida de sí mismo como sujeto y, por cierto, como sujeto artísticamente creador, vive con cierta calma, seguridad y consecuencia; si pudiera salir, aunque sólo fuese un instante, fuera de los muros de esa creencia que lo tiene prisionero, se terminaría en el acto su "conciencia de si mismo". Le cuesta trabajo reconocer ante sí mismo que el insecto o el pájaro perciben otro mundo completamente diferente al del hombre y que la cuestión de cuál de las dos percepciones del mundo es la correcta carece totalmente de sentido, ya que para decidir sobre ello tendríamos que medir con la medida de la percepción correcta, es decir, con una medida de la que no se dispone,3

Como podemos ver en el párrafo superior, el modo de conocer, de percibir la realidad no es único y absoluto, Nietzsche lo muestra aquí a través de la comparación entre el hombre y dos especies del género no humano. Pero, ¿acaso no podemos sostener lo mismo entre distintas grupos humanos? El autor está otra percepción de la realidad diferente a la del hombre, la que puede tener el pájaro o el insecto, pero no es menos cierto que esta otra forma de percibir, y por ende, de conocer, no es la misma en la totalidad de la especie humana. No

\footnotetext{
${ }^{2}$ Pretendo aclarar que a lo que me refiero con teorías absolutas o cerradas de la verdad (las nombraré indistintamente), son las distintas gnoseologías que las filosofía occidental en su transcurrir nos ha proporcionando, teniendo en cuenta, tan sólo, un contexto sociocultural determinado, y cuya pretensión de universalización ha sido total. ${ }^{3}$ Ibid, p 29
} 
considero necesario acudir a otras especies vivas para explicar lo que dentro del mismo grupo de humanos podemos advertir con claridad. Distintos grupos humanos, culturalmente diferentes, perciben realidades distintas dependiendo de sus vivencias, contextos, en definitiva, de su habitus. Este concepto desarrollado por Bourdieu, nos permite ver como debido al mismo se forman esquemas a través de los cuales los individuos perciben la realidad, aprecian estas u otras cosas, dividen o categorízan de esta u otra manera, evalúan conforma a su determinado habitus, etc.

"Los condicionamientos asociados a una clase particular de condiciones de existencia producen habitus, sistemas de disposiciones duraderas y transponibles, estructuras estructuradas predispuestas a funcionar como estructuras estructurantes, es decir, en tanto que principios generadores y organizadores de prácticas y representaciones que pueden estar objetivamente adaptadas a su fin sin suponer la búsqueda consciente de fines y el dominio expreso de las operaciones necesarias para conseguirlos, objetivamente 'reguladas' y 'regulares' sin ser para nada el producto de la obediencia a reglas, y siendo todo esto, objetivamente orquestadas sin ser el producto de la acción organizadora de un jefe de orquesta"4

\subsection{Wittgenstein}

Dejemos de momento a Nietzsche y vayamos a jugar con el lenguaje y con Wittgenstein. En sus Investigaciones filosóficas es de sobra conocido como traiciona por culpa de la práctica su anterior teoría del lenguaje del Tractatus, veamos ahora como a través de esta obra podemos advertir el cuestionamiento que vengo sosteniendo de las teorías de la verdad cerradas, y mi defensa de una pluralidad de modos de conocer. Continuando con la idea de "modos de" que hemos visto que favorece el habitus, todo juego de lenguaje está relacionado también con un determinado modo de, en concreto, un modo de una forma de vida:

"La expresión "juego del lenguaje” debe poner de relieve aquí que hablar el lenguaje forma parte de una actividad o de una forma de vida"

Y no debemos olvidar aquí, en lo que nos atañe en cuanto al conocimiento, que el pensamiento depende del lenguaje ${ }^{6}$ (volveremos a

\footnotetext{
${ }^{4}$ Bourdieu ,El sentido práctico, Taurus, Madrid, 1991, p 92

${ }^{5}$ Wittgenstein, Investigaciones filosóficas, UNAM/Crítica, Barcelona, 1988, p 39
} 
esta idea después), de modo que los distintos lenguajes posibles puedan dar cuenta de un pensamiento que se corresponda a ellos. De ahí deviene la aparente incomprensión de otras lenguas, con las que compartimos un contextos cultural similar (que salvamos con la traducción) o con las que no (cuyo forma de solucionarlo no depende sólo de la intertextualidad, sino de un conocimiento más profundo, en palabras de Geertz, de una "descripción densa"7). Wittgenstein soluciona este dilema, de otra forma parecida, adivinando la interpretación de esa lengua, es lo que hace el antropólogo hermeneuta Geertz sin duda, adivinar interpretando:

“Quien llega a un país extraño aprenderá a veces el lenguaje de los nativos por medio de explicaciones extensivas que ellos le den; y a menudo tendrá que adivinar la interpretación de estas explicaciones y adivinar unas veces correctamente y otras erróneamente" ${ }^{, 8}$

En la idea de juegos de lenguaje podemos advertir la presencia de una diversidad lingüística más que evidente. Lo que vengo a resaltar aquí es la relación que estos juegos lingüísticos tienen con la capacidad intelectiva, una relación que sin duda está claramente mediada por una realidad fueran del intelecto humano, por la realidad exterior que se muestra y se impone de diversas maneras en diversos grupos culturales:

"El pensamiento, el lenguaje, nos parece ahora como el peculiar correlato, o figura, del mundo. Los conceptos: proposición, lenguaje, pensamiento, mundo, están en serie uno tras otro, cada uno equivalente a los demás. (¿Pero para que han de usarse ahora estas palabras? Falta el juego del lenguaje en el que han de aplicarse.)"9

Falta, por tanto, el contexto cultural en el que aplicar este u otro juego del lenguaje, con sus reglas y sus técnicas, y será este el que precisamente lo dotará de sentido, de significado, en definitiva, de coherencia interna. Y puesto que el significado de la palabra es su uso en el lenguaje, deberíamos entender pues, que hay tantos significados como juegos del lenguaje. Por tanto, ante la realidad que nos muestra Wittgenstein en el siguiente párrafo, ¿cuál es el significado?:

\footnotetext{
${ }^{6}$ Sigo aquí la teoría del lenguaje de Benjamín Lee Whorf.

${ }^{7}$ Véase La interpretación de las culturas de Clifford Geertz

${ }^{8}$ Investigaciones filosóficas o.c., p 49

${ }^{9}$ Ibid., p 117
} 


\begin{abstract}
"Es, naturalmente, imaginable que en una tribu que no conoce el juego dos personas se sienten ante a un tablero de ajedrez y ejecuten los movimientos de una partida de ajedrez; e incluso con todos los fenómenos mentales concomitantes. Y si nosotros lo viésemos, diríamos que juegan al ajedrez. Pero imagínate ahora una partida de ajedrez traducida ahora mediante ciertas reglas en una serie de acciones que no estamos habituados a asociar con un juego digamos una preferencia de gritos y patadas con los pies. Y ellos dos deben ahora, en vez de jugar a la forma de ajedrez que nos es familiar, gritar y dar patadas; y justamente de modo que ese proceso pueda traducirse mediante reglas apropiadas en una partida de ajedrez"
\end{abstract}

Me parece suficientemente esclarecedor con este ejemplo, que el principal problema esta en la formulación de la pregunta antes planteada, planteando la singularidad del significado. La pregunta sería correcta si se plantease siendo conscientes de la restricción de la misma a un grupo "x", pero no a la totalidad de los grupos. En el ejemplo de Wittgenstein podemos advertir dos significados totalmente coherentes con el marco cultural que los conforma, y con las reglas implitas -que no explicitas-, que toda actuación humana tiene (aunque la desconozcamos):

"Seguir una regla es análogo a: obedecer una orden. Se nos adiestra para ello y se reacciona a ella de determinada manera. ¿Pero qué pasa si uno reacciona así y otro de otra manera a la orden y al adiestramiento? ¿Quién está en lo correcto? Imagínate que llegas como explorador a un país desconocido con un lenguaje extraño. ¿Bajo qué circunstancias dirías que la gente de allí da órdenes, entiende órdenes, obedece, se rebela contra órdenes, etc.? El modo de actuar humano común es el sistema de referencia por medio del cual interpretamos un lenguaje extraño",11

Conectando esta diversidad de significados (desde mi postura, igualmente verdaderos ambos dentro de su lógica cultural) con los distintos modos de conocimiento según la contextualización del mismo con el entorno sociocultural, observemos otro ejemplo de Wittgenstein:

"Cuando los niños juegan al ferrocarril, su juego está conectado con su conocimiento del ferrocarril. Pero los niños de una tribu que desconocen el ferrocarril podrían haber tomado ese juego de otros y jugarlo sin saber que con él se imitaba algo. Podría decirse que el juego no tiene para ellos el mismo sentido que para nosotros",12

\footnotetext{
${ }^{10}$ Ibid., pp 201-203

${ }^{11}$ Ibid., p 205

${ }^{12}$ Ibid., p 239
} 
¿Qué forma de jugar al ferrocarril es la correcta, y por qué?, debido al conocimiento real del primer grupo de niños, su forma de jugar estará relacionada firmemente con su conocimiento, sin embargo, el segundo grupo de niños lo más probable es que relacionen su juego con una realidad diferente aunque más cercana a su contexto, y con toda probabilidad, si pudiésemos preguntarles el por qué de esa manera de jugar, veríamos que no es para nada descabellada la respuesta (de esto es de lo que precisamente se encarga la antropología, de desenmarañar el significado interno de la acción social de individuos que por lejanía cultural no alcanzamos a comprender su agencia).

\subsection{El tiempo como ejemplo}

Aproximándonos a las teorías de estos dos autores se me muestra cada vez más evidente que de ellas se puede desprender una claro relativismo que me cuesta mucho apreciar en otras gnoseologías como, por ejemplo, en la Crítica de la Razón Pura de Kant, el que en lo que se refiere a la estética trascendental, entiende como el espacio y el tiempo dos principio posibilitantes del conocimiento. Aquí entiende el tiempo como:

"la forma (el modo de funcionar) de los sentidos internos (y por lo tanto la forma de todos los datos sensibles internos en la medida en que sean conocidos por nosotros),

Sin embargo, esta forma claramente occidental de entender el tiempo difiere mucho de la forma en que entiende el tiempo el indio hopi, por lo que considero que deberíamos humildemente reconocer la falta de universalidad de esta teoría de Kant, o a lo sumo, restringirla a un grupo sociocultural que comportante esta estructuras posibilitantes de conocimiento. Siendo conscientes que la diversidad cultural, también conlleva otro tipo de diversidad lingüística y gnoseológica:

"Creo que es gratuito suponer que un hopi que sólo conoce su lengua y las ideas culturales de su propia sociedad, tiene las mismas nociones que nosotros sobre espacio y tiempo, nociones que a menudo se suponen son

${ }^{13}$ Reale y Antiseri, Historia del pensamiento filosófico y científico, vol. II, Herder, Barcelona 2005, p 738 
intuiciones universales. En particular, un hopi no tienen la noción o intuición general de TIEMPO como un continuum que transcurre uniformemente y en el que todo lo que hay en el universo marcha a un mismo paso, fuera de un futuro, a través de un presente y procedente de un pasado, o, para cambiar de imagen, en el que el observador es llevado constantemente, por la corriente de la duración, alejándolo el pasado, hacia el futuro"14

Y como no hay dos sin tres, otro ejemplo es el de los trobiandeses que estudio Malinowski. Peacock nos lo cuenta así:

"Nos han enseñado está forma de pensar en el colegio, donde, haciéndonos pasar por una serie de estadios, se nos conduce hasta la graduación; en los refranes, que nos dicen que el tiempo es oro, que todo lo trae $\mathrm{y}$ todo lo lleva, y que hay que aprovecharlo y no desperdiciarlo. Nos hemos criado pensando en el tiempo de esta forma lineal. Lo hacemos sin recapacitar sobre ello. Damos por hecha esa forma de pensar. Antropólogos como Hall nos ensañan que no todo el mundo piensa de ese modo. Parece que los habitantes de las islas Trobiand del Pacífico occidental partían de otros presupuestos. Se dice que, a diferencia del triunfador esforzado, a los trobiandeses no les preocupaban especialmente las interrupciones o que ni siquiera consideraban que un obstáculo en la realización de una tarea constituyera una interrupción. Para ellos el tiempo no era tanto una línea sobre la que uno se mueve como un charco en el que uno se sienta, chapotea o se revuelca. Los trobiandeses imaginaban el tiempo como algo carente de dirección, no como una línea que indica una trayectoria", 15

Más adelante profundizaremos con algo más de detalle en esta relación de las distintas realidades externas con los diversos modos intelectivos internos y la configuración del lenguaje que los media. Pero creo conveniente antes, introducir algunas precisiones sobre la antropología social y cultural (desde la cual organizado el discurso que aquí presento), así como algunos aspectos metodológicos propios de la disciplina, que mostraran otro modo de conocer alejando del abstractivo de la disciplina filosófica, y con un carácter en parte puramente práctico que es el que fundamental la posterior teoría antropológica. La exposición que haré, advierto que es bastante escueta debido al espacio y al tiempo (entiéndase kantianos) que aquí me trae.

\footnotetext{
${ }^{14}$ Whorf, Lenguaje, pensamiento y realidad, Barral, Barcelona, 1971, p 73

${ }^{15}$ Peacock, La lente antropológica, Alianza, Madrid, 2005, pp 29-30
} 


\section{3. ¿Qué es eso de la antropología?}

\subsection{Precisando el concepto}

Lo primero de todo, advertir que en estas páginas al hablar de antropología me estaré refiriendo única y exclusivamente a la antropología social y cultural. Es difícil encontrar en los manuales de antropología definiciones de qué es la antropología, en este, como en otros aspectos, la prudencia del antropólogo está más que presente. Apuntaré aquí algunas de ellas, para que nos vayamos haciendo una idea de lo que es. Kottak (profesor del departamento de Antropología en Michigan) en su famosísimo manual de Antropología, la describe de la siguiente manera:

"La antropología cultural estudia la sociedad y la cultura humana, describiendo y explicando, analizando e interpretando las similitudes y diferencias culturales"

Radcliffe-Brown, la figura por excelencia de la antropología social británica, que dio a conocer como el funcional estructuralismo, la define así:

"Concibo la antropología social, como el estudio teórico comparativo de formas de vida social entre los pueblos primitivos" $" 17$

Veamos una última definición, y pasaremos a comentarlas y ponerlas en relación, buscando una síntesis que nos ayude a dibujar el concepto. Esta vez, le dejaremos la palabra al maestro Lévi-Strauss:

“QQué es pues la antropología? Por el momento, limitémonos a decir que ella deriva de una cierta concepción del mundo y de una manera original de plantear los problemas, cosas ambas descubiertas 'con ocasión' del estudio de fenómenos sociales que no necesariamente son más simples (como tan a menudo se tiende a suponer) que aquellos que tienen por teatro la sociedad del observador, pero que -en razón de las grades diferencias que presentan en comparación con estos últimos- ponen de manifiesto ciertas 'propiedades generales' de la vida social que el antropólogo toma como objeto de estudio",18

\footnotetext{
${ }^{16}$ Kottak, Antropología cultural, McGraw-Hill, Madrid, 2006, p 8

Radcliffe-Brown, Estructura y función en las sociedades primitivas, Península, Barcelona, 1974, p 12

${ }^{18}$ Lévi-Strauss, Antropología estructural, Eudeba, Buenos Aires, 1984, p 311
} 
A continuación entresacaré algunos términos o ideas que comentar. Como vemos más arriba, un término fundamental en el estudio de la disciplina es el concepto de $\underline{\text { cultura }}^{19}$, de ahí que muchas veces se hable de antropología como la ciencia de la cultura. Este término, a veces como en el caso de Lévi-Strauss no está explicitado, pero es notable que está ahí latente. Podemos decir, que el estudio de la antropología es el hombre en su condición de ser cultural (o social) ${ }^{20}$. ¿Y qué es la cultura?, citaré sin más la definición más conocida que es la Tylor:

"La cultura o civilización, en sentido etnográfico amplio, es aquel todo complejo que incluye el conocimiento, las creencias, el arte, la moral, el derecho, las costumbres y cualesquiera hábitos y capacidades de la sociedad"21

Si pretendemos tener alguna característica lo más definitoria posible de la disciplina, considero que lo más oportuno es distinguir su carácter holístico. Su pretensión de abordar la realidad a estudiar en su conjunto, no separadamente, sino de una forma relacional de las partes con el todo:

"Esta amplia perspectiva, a veces denominada 'holística', quizá sea, en sí misma, el rasgo más sorprendente de la antropología. Cualquiera que sea la definición de antropología que se elija, habrá que subrayar que esta es una disciplina para comprender a la humanidad en su muy diversas facetas, es decir, holísticamente" 22

En la definición de Radcliffe-Brown hace referencia a los primitivos, en tanto otro. Es normal asociar al antropólogo con la tribu, los salvajes. Pero hace tiempo ya que los antropólogos salieron de la selva para investigar en las sociedades industrializadas. El otro al que se estudia, ya no lleva taparrabos, en la actualidad es más usual que nuestro investigado lleve corbata a que se cubra con taparrabos. Así, algunas de las investigaciones de la disciplina recientes en nuestras universidades españolas están teniendo como objeto de estudio, prácticas de anoréxicas

\footnotetext{
${ }^{19}$ Para un mejor abordaje del término, Antropología de Kluckhohn.

${ }^{20}$ En la definición de Kottak se hace referencia a la antropología cultural, mientras que en la de Radcliffe-Brown se refiere a la antropología social. Quisiera aclarar que esta distinción, es de carácter geográfico y por cuestión de predilección. Gran Bretaña utilizó el término "social", y Estados Unidos el término "cultural".

${ }^{21}$ Tylor, "La ciencia de la cultura", en: Kahn, J. S. (comp.): El concepto de cultura. Anagrama. Barcelona. 1995, vol. I, 1

${ }^{22}$ La lente antropológica o.c., p 37
} 
nerviosas, la memoria en los familiares en exhumaciones de cadáveres represaliados en la época franquista, adopciones en familias homoparentales, etc., como vemos, el sujeto de estudio no el mismo que en los comienzos de la disciplina. El otro, ahora cada vez está más próximo a nosotros, ya no es necesario cruzar charcos para hacer etnografía.

Podemos intentar una síntesis diciendo que, la antropología es un tipo de conocimiento holístico, de carácter práctico en primer lugar, desarrollando una teoría después, que tiene como foco principal al hombre en tanto ser cultural, en sus diversas manifestaciones y aspectos, y se caracteriza por un metodología específica, la cual le otorga validez su carácter científico.

\subsection{Líneas históricas}

Pero antes, utilizaré unas líneas para permitirme hacer una síntesis muy concisa sobe la historia de la antropología. Señalaré en el siguiente cuadro las principales escuelas y autores.

\begin{tabular}{|c|c|c|}
\hline $\begin{array}{l}\text { Evolucionismo } \\
(1840-1890)\end{array}$ & Tylor, Morgan, Frazer & \\
\hline $\begin{array}{l}\text { Difusionismo } \\
(1880-1940)\end{array}$ & Wissler, Kroeber, McNeill & \\
\hline \multirow[t]{3}{*}{ Funcionalismo } & $\begin{array}{l}\text { Escuela sociológica francesa (1920- } \\
\text { 1930) }\end{array}$ & $\begin{array}{c}\text { Durkheim, } \\
\text { Mauss, Levy-Bruhl }\end{array}$ \\
\hline & $\begin{array}{l}\text { Funcionalismo británico }(1920- \\
\text { 1930) }\end{array}$ & Malinowski \\
\hline & $\begin{array}{l}\text { Estructural-funcionalismo } \\
\text { 1950) }\end{array}$ & \begin{tabular}{l}
\multicolumn{2}{c}{ Radcliffe- } \\
Brown, Evans- \\
Pritchard
\end{tabular} \\
\hline \multirow[t]{3}{*}{ Culturalistas } & $\begin{array}{l}\text { Particularismo histórico (1899- } \\
\text { 1940) }\end{array}$ & Boas \\
\hline & Superorganicista (1915-1920) & Kroeber \\
\hline & Cultura y personalidad (1920-1960) & Benedict, Mead \\
\hline Estructuralismo & Lévi-Strauss (1960-1970) & \\
\hline Simbolismo & Douglas, Turner (1950-1970) & \\
\hline \multirow[t]{2}{*}{ Materialistas } & Neoevolucionismo (1940-1950) & White \\
\hline & Ecología cultural (1940-1950) & Steward \\
\hline
\end{tabular}




\begin{tabular}{|c|l|l|}
\hline \multirow{2}{*}{} & Funcionalismo cultural & Rappaport \\
\cline { 2 - 3 } & Materialismo cultural (1970) & Harris \\
\hline Posestructuralismo & Nueva Etnografía (1980) & Goodenough \\
\cline { 2 - 3 } & Interpretativismo (1980) & $\begin{array}{c}\text { James } \\
\text { Fernández }\end{array}$ \\
\cline { 2 - 3 } & Posmodernismo (1980) & Geertz \\
\hline
\end{tabular}

El evolucionismo piensa en la cultura en constante evolución, así se pueden distinguir culturas menos evolucionadas que otras. Entendiendo así Morgan tres períodos distintos (salvajismo, barbarie y civilización) en tanto sus estructuras de parentesco, y Lévy-Bruhl en términos de la estructura mental humana (distinguiendo mentalidad primitiva y mentalidad moderna).

Los difusionistas, explican el desarrollo histórico en términos de la difusión de rasgos culturales desde un punto de origen a otros de llegada, entendiendo así la adopción de rasgos por imitación en contactos cultural tales como las migraciones.

La teoría del funcionalismo trabaja teniendo en cuenta que todo elemento cultural se entiende como orientado a satisfacer unas necesidades determinadas. Todo sistema cultural está interrelacionado para ese fin. Su mayor representante Malinowski lo define de la siguiente manera:

"Esta clase de teoría aspira a explicar las realidades Antropológicas en todos sus niveles de desarrollo por su función, por la parte que juegan dentro del sis-tema integral de la cultura, por la manera como se hallan relacionadas unas con otras dentro del sistema y por la forma en que este sistema se halla vinculado en su contorno físico" 23

El particularismo histórico de Boas hace referencia a la investigación exhaustiva de cada cultura particular, tomando como bandera el relativismo cultural. Inicia una nueva orientación que continuarán Benedict y Mead en la corriente de cultura y personalidad, acentuando que los modos culturales diferentes, no sólo se deben a situación fisiológicas distintas, también tiene en cuenta los factores psicológicos.

${ }^{23}$ Malinowski, La vida sexual de los salvajes del Noroeste de la Melanesia, Morata, Madrid, 1971, p 34 
La corriente materialista supone un neoevolucismo, relacionado con la teoría ecológica cultural, entendiendo que la cultura es una fábrica de energía, y así la cultura avanza en la medida en que la energía aumenta.

El estructuralismo de Lévi-Strauss, se basa en el estudio de diversos pueblos para conformar aquello que es común y universal al género humano, las categorías universales del pensamiento que sirvan para comprender a hombre. Cada sociedad tiene sus propios códigos, y un conocimiento comparado de los mismos nos permite desvelar un código universal, porque todos son formas de leyes universales en que actúa el inconsciente: por ejemplo, la ley de prohibición del incesto.

La escuela simbólica presta atención a la dimensión simbólica de las prácticas humanas, símbolos culturales que sirven para explicar las conductas de un determinado grupo social, manteniendo un sistema de significados compartidos. Esta escuela está relacionada con la interpretativista o hermenéutica, de la que es figura importante Geertz.

\subsection{Metodología}

Quizá sea algo más peculiar de lo que es la disciplina antropológica, el método que utiliza, lo que propiamente lo distingue de otras ciencias sociales, lo que le da rigor a los propios resultados. El principal rasgo es el famoso trabajo de campo, que tantas alegrías y tormentos provoca al investigador. Este aspecto metodológico es el que más nos interesa, ya que es el que conlleva una pretensión de conocimiento determinado.

El modo de conocimiento de la realidad social propio de la antropología es la etnografía. Un método de conocimiento de carácter cualitativo. Me atrevería a sostener que es quizá el método lo que en gran medida define a la disciplina antropológica. Así es usual definir al antropólogo por aquello que hace, la etnografía, cuya parte fundamental es el trabajo de campo. Hammersley y Atkinson, definen así a la etnografía:

"Entendemos el término como una referencia que alude principalmente a un método concreto o a un conjunto de métodos. Su principal característica sería que el etnógrafo participa, abiertamente o de manera encubierta, en la vida diaria de las personas durante un período de tiempo, observando lo que 
sucede, escuchando qué se dice, haciendo preguntas; de hecho, haciendo acopio de cualquier dato disponible que sirva para arrojar un poco de luz sobre el tema en que se centra la investigación",24

Para Malinowski, el fundamental la explicitación de la metodología en todo trabajo de investigación para poder dar cuenta de la rigurosidad del mismo, y es lo que hace en la introducción de Los argonautas del Pacífico occidental, exponiendo algunas bases sobre las que se erige posteriormente la teoría metodológica etnográfica:

"Sólo obtendremos resultados satisfactorios si aplicamos paciente y sistemáticamente cierto número de reglas de sentido común y los principios científicos demostrados, y nunca mediante el descubrimiento de algún atajo que conduzca a los resultados deseados sin esfuerzo ni problemas. Los principios metodológicos pueden agruparse bajo tres epígrafes principales; ante todo, el estudio debe albergar propósitos estrictamente científicos y conocer las normas y los criterios de la etnografía moderna. En segundo lugar, debe colocarse en buenas condiciones para su trabajo, es decir, lo más importante de todo, no vivir con otros blancos, sino entre los indígenas. Por último, tiene que utilizar cierto número de métodos precisos en orden a recoger, manejar y establecer pruebas" 25

Hay una referencia al sentido común en el fragmento que he extraído de la obra de Malinowski que puede llevar a pensar en la falta de cientificidad de los estudios etnográficos. Quisiera aclarar este comentario para hacer ver que esto más que un impedimento, es una ayuda en el proceso investigador. En otras ciencias puramente cuantitativas, en las que el objeto a estudiar no es un sujeto, podemos decir que el sentido común no tienen un papel tan fundamental (y no tiene por que tenerlo) como en la antropología. Pero como ya hemos visto, ésta estudia al ser humano holísticamente, y en su investigación, el sujeto investigador y el sujeto-objeto investigado, comparten una misma realidad que es la subjetividad, y es propiamente el reconocimiento de que el investigado no es un objeto sino un sujeto, el que me sitúa en un plano diferente en el que el contacto personal y la confianza mutua juegan papeles principales. Y es por ello, que en este tipo de

\footnotetext{
${ }^{24}$ Hammersley y Atkinson, Etnografía. Métodos de investigación, Paidos, Barcelona, 2009, p 15

${ }^{25}$ Malinowski, Los argonautas del Pacífico occidental, Península, Barcelona, 2001, pp 45-46
} 
investigación el sentido común puede ser una técnica más en nuestro proceso investigador.

Como podemos apreciar el trabajo de campo etnográfico requiere de tiempo en es espacio en el que están nuestros sujetos a investigar, para ver las cosas "desde dentro". Es usual que las investigaciones en el terreno, sea cual fuera este, sean de periodos relativamente largos en el tiempo, y tras un alejamiento temporal del campo, para ver las cosas, esta vez, "desde fuera", reflexionar, analizar el proceso en el que se encuentra la investigación, para poder volver otra vez más si fuese necesario. El ejemplo de Malinowski, de estar 5, 9 y 12 meses en campo es más que relevante de este hecho. Pero para estudiar la cultura ajena es necesario, en cierto modo, olvidarse de la propia y zambullirse de lleno la otra para de verdad comprender, vivir, y sentir, logrando aspirar a aquello que Malinowski llama el punto de vista del nativo:

"La supuesta plena integración, la conversión a otra cultura [...] no se intenta sino para lograr un conocimiento profundo, un acceso al significado de los comportamientos, y para realizar luego un relato dirigido a otros acerca de cómo son, cómo viven, piensan, ven el mundo las gentes entre las que se ha socializado el investigador, 26

\subsubsection{Técnicas}

Como hemos visto en la definición de Hammersley y Atkinson, hacen referencia a técnicas para la consecución del fin propuesto, conseguir el punto de vista del nativo. Estas técnicas no son un cajón cerrado de herramientas que tenga que usarse necesariamente, de hecho cada vez más estudios etnográficos están utilizando técnicas de corte cuantitativo, más propias de la sociología como la estadística, como apoyo a las más puramente etnográficas, que son cualitativas. Pasaremos a ver algunas de estás técnicas que la etnografía usa habitualmente. La observación participante y la entrevista en profundidad o abierta son las dos fundamentales, por ello las tendré más en cuenta.

\footnotetext{
${ }^{26}$ Velasco y Díaz de Rada, La lógica de la investigación etnográfica, Editorial Trotta, Madrid, 2006, p 27
} 


\section{Observación participante}

Será Malinowski en 1914 el que sentará unas bases en antropología al producir una ruptura con los antropólogos anteriores de "sillón", adentrándose "en campo", y participando directamente de la realidad observada que anteriormente no se había tenido en cuenta. Podemos decir que a modo de testigo de lo que allí acontece.

Podemos distinguir varios niveles de participaciónobservación. Por una parte, tenemos la "participación total", en la que el observador forma parte del grupo a investigar, o intenta formar parte, sin rebelar su rol de investigador. Esto cuenta a su favor con que se evita las cuestiones relativas al "acceso a campo" como investigador, y obtiene una información desde dentro, pero en su contrapartida corre el riesgo de ser descubierto y que su investigación termine de una forma no deseada. Por otra parte, la "observación total" tiene en su favor que minimiza los problemas de negación de acceso, porque no se conoce las pretensiones del investigador, pero tiene en su contra la falta de accesibilidad a una gran parte de información relevante para cualquier estudio cultural. Con cualquiera de estas dos perspectivas, es difícil hacer un trabajo de campo riguroso. Lo ideal es encontrar ese punto intermedio entre la observación y la participación, que se conoce como "observación participante". Es a través de la misma como se observa y se forma parte a la vez de los hechos y sucesos que estamos estudiando en la vida de un grupo determinado. Se participa aquello que estamos observando. Berreman define así la observación participante:

"La observación participante se refiere a la práctica que consiste en vivir entre la gente que uno estudia, llegar a conocerlos, a conocer su lenguaje y sus formas de vida a través de una intensa y continua interacción con ellos en sus vida diaria. Esto significa que el etnógrafo conversa con la gente, trabaja con ellos, asiste a sus funciones sociales y rituales, visita sus casas y les invita a la suya, es decir, está presente en tantas situaciones como sea posible, aprendiendo a conocerles en tantos ambientes y desde tantas facetas como pueda" 27

Mediante esta técnica de observación participante se accede a datos que de otra manera no sería posible, como son los que Malinowski

\footnotetext{
${ }^{27}$ Berreman, "Ethnography: method and product", en: J. A. Clifton (ed), Introduction to cultural anthropology: seáis in the socope and methods or the science of man. Boston. Houghton Mifflin C., 968, p 337
} 
denomina "imponderables de la vida real", aquellos detalles íntimos de la vida cotidiana que son tan naturales dentro de la cultura a estudiar que pasan en gran medida desapercibidos como elementos específicos de la propia cultura:

"Llamémosles los imponderables de la vida real. Aquí se engloban cosas como la rutina del trabajo diario de los individuos, los detalles del cuidado corporal, la forma de tomar los alimentos y de prepararlos, el tono de la conversación y la vida social que se desarrolla alrededor de los fuegos de la aldea, la existencia de fuertes amistades o enemistades y de corrientes de simpatía y antipatía entre la gente, la manera sutil pero inconfundible en que las vanidades y ambiciones personales se reflejan en el comportamiento del individuo y las reacciones emocionales de los que les rodean"28

El observador debe describir después aquello que ha estado observando y de lo cual ha participado en un registro, que con posteridad le servirá para acceder a la información con la precisión y frescura del primer día, sin que el paso del tiempo haya borrado determinados datos de su memoria. Los registros deben realizarse de forma sistemática, lo antes posible ya que es entonces cuando aun está reciente todo lo acaba de acontecer, aunque esta labor, junto a la trascripción de las entrevistas (que a continuación veremos) son las más tediosas a realizar. Estos registros forman un diario de campo, que se va completando con los diversos registros realizados durante el proceso de trabajo de campo, y que, a su vez, son la fuente en la que el investigador en la posterior elaboración analítica se puede fundamentar.

\section{b) Entrevistas}

En el trabajo de campo etnográfico, no toda la información es observable por el investigador, hay distintas clases de información a la no podemos acceder simplemente con "estar ahí". Para esa otra información no observable, la otra técnica principal (junto a la observación participante) es la entrevista. La entrevista puede ser de carácter más informal, en encuentros casuales en la calle por ejemplo, o entrevistas formales abiertas, donde el entrevistador prepara y acuerda con el entrevistado un día, lugar y hora donde llevarla a cabo. Así definen Taylor y Bogdan estas entrevistas:

${ }^{28}$ Los argonautas del Pacífico occidental o.c., pp 66-67 
"reiterados encuentros cara a cara entre el investigador y los informantes, encuentros estos dirigidos hacia la compresión de las perspectivas que tienen los informantes respecto de sus vidas, experiencias o situaciones, tal como las expresan con sus propias palabras",29

Esta entrevista no es un cuestionario cerrado donde el entrevistador pregunta y el entrevistado responde. Al contrario, lo ideal en la entrevista abierta es que apenas tenga que intervenir el entrevistador, más allá de lo necesario, dejando al entrevistado construir un discurso lo más libre posible. Los antropólogos no van con una serie de preguntas dispuestos a dispararlas para que todas ellas sean contestadas en lo que dura la entrevista, al contrario, preparan la entrevista teniendo cuenta aquello que quieren obtener de la misma, pero sin llevar formuladas de antemano las preguntas tal cual. Podemos decir que en la entrevista antropológica no hay preguntas hay temas. El modo de preguntar es no indirecto, abierto, para que el entrevistado no se limite a un "sí" o un "no", sino que tenga que elaborar una argumentación a través de la cual obtener más información que la se da (por ejemplo, el nivel social del entrevistado). La entrevista, tal y como la entiendo la antropología, se parece más a una conversación entre iguales, que a un cuestionario. En ocasiones también pueden hacerse entrevistas grupales.

La entrevista se registra bien en una grabadora de audio, bien en videocámara, con la finalidad de trascribirla después literalmente (con sus énfasis, dudas, silencios, risas, etc.) y ser lo más fiel al discurso obtenido. Con anterioridad se le informa al entrevistado de la total confidencialidad de su discurso que no será escuchado por nadie, preservando su identidad. Una vez trascrita la entrevista, se procede igual que con los registros al análisis de los mismos.

Otras técnicas que dependen de entrevistas son las historias de vida y las redes sociales de Internet, que comentaré brevemente. Las historias de vida ${ }^{30}$ son el resultado de varias entrevistas realizadas a una misma persona con la que se pretender reconstruir la memoria de esta persona, que refiere su vida en un periodo histórico determinado y en una comunidad precisa. La historia de vida, aunque puedan parecerse a las

\footnotetext{
29 Taylor y Bogdan, Introducción a los métodos cualitativos de investigación, Barcelona, Paidos, 1992, p 101

${ }^{30} \mathrm{Si}$ se pretende una mayor acercamiento a esta técnica pueden consultarse las historias de vida de Olga Visuales y Fernando Sáez (practicantes de BDSM) y Oriol Romaní (de un drogadicto), citadas en la bibliografía.
} 
biografías o autobiografías, no es lo mismo, se diferencian que las historias de vida surgen a lo largo de un proceso investigador, y cuya finalidad es conocer, a través de este testimonio concreto, las claves para comprender en contexto y sociedad al que pertenece el informante:

"Obtener relatos de vida de actores que ejemplifiquen bien figuras, roles y enclaves característicos de la vida en la sociedad estudiada nutre es estudio de una etnografía honda en el tiempo y densa en la vida social que ayuda a identificar una imagen más rica, dinámica y realista del grupo que observamos",31

Las redes sociales de Internet, tales como foros o chat, son un medio a través del cual entrevistar informantes, con la ventaja de que el texto al quedar almacenado en la memoria, no requiere de trascripción, y está listo para su análisis. Es necesario tener en cuenta los sesgos propios de estos medios, y tomar su información con la suficiente prudencia. Este medio tiene, como todos, ventajas e inconvenientes. El principal inconveniente en la falta de contacto físico y visual con el interlocutor, la falta de rapport en la conversación. Y la ventaja que yo encuentro es tener la posibilidad de entrevista a alguien que por motivos ajenos a la investigación no puede ser físicamente entrevistado ${ }^{32}$.

\section{c) Método genealógico}

El método genealógico es una técnica que hoy en día está más en desuso, aunque en los orígenes de la disciplina era indispensable, esto es debido a que ahora, cada vez menos, se estudian sociedades no industriales, donde es totalmente necesario el abordaje genealógico para entender parentescos, líneas de filiación, derechos de propiedad, alianzas sociales, etc., todas la multiplicidad de cuestiones que de las redes sociales de parentesco se derivan.

\footnotetext{
${ }^{31}$ Sanmartín Arce, "El trabajo de campo", en: Lisón Tolosana, C. (ed.): Introducción a la antropología social y cultural, Akal, Madrid, 2007, p 73

${ }^{32}$ Aclarar que todas las entrevistas a través de Messenger que yo he realizado han sido con un conocimiento anterior físico y personal de la persona en cuestión. Y considero que este es requisito indispensable para que el resultado de la entrevista puede ser utilizado analíticamente.
} 


\section{d) Otras técnicas}

Otras técnicas ya no tan específicas de la disciplina, pero que en ocasiones pueden ser utilizadas dependiendo de las necesidades, son los grupos de discusión, las técnicas fotográficas y/o audiovisuales, las encuestas y todo documento de interés pertinente. De antemano sostener que aunque la antropología como tal tiene un corte puramente cualitativo, depende del investigador integrar técnicas de carácter cuantitativo en el proceso de investigación. Actitud que yo apoyo, en tanto en cuanto, sea permitente y se utilice junto con otras técnicas cualitativas, en pro de conseguir los objetivos propuestos.

\subsubsection{Mirada antropológica}

Hasta aquí he intentando poner de relieve que una parte sustancial de la investigación antropológica es la metodología que utiliza. Pero, toda persona que haga investigación aferrándose a esta etnografía, ¿está haciendo realmente antropología? Para algunos investigadores puede que esto sea más que suficiente para que su obra sea entendida como tal. Sin embargo, no quiero pasar por alto, lo que considero igualmente importante que la metodología, que es lo que María Isabel Jociles llama "mirada antropológica":

"Lo que permite aceptar una investigación como antropológica no es el recurrir a un procedimiento, a un campo, a una técnica o conjunto de técnicas determinado, sino el uso que de ellas hace un investigador que se ha formada un 'mirada' que consideramos antropológica y que las sitúa en una situación etnográfica. Y, por supuesto, si la 'especificidad' de una investigación está en la 'mirada', en el 'enfoque', lo mismo cabe predicar de su calidad"33.

Por lo tanto, como comenta la autora, atendiendo a este hecho, podemos considerar que en el proceso de investigación el investigador es una herramienta más en este juego de conocimiento. No sólo la metodología etnográfica, sino él mismo como instrumento a través del cual conocer de una manera determinada. Además, esta mirada

\footnotetext{
33 Jociles, "Las técnicas de investigación en antropología: mirada antropológica y proceso etnográfico" Gazeta de antropología, 15 (1999) http://www.ugr.es/ pwlac/G15_01MariaIsabel_Jociles_Rubio.html
} 
está presente durante todo el proceso de investigación, e incluso me atrevería a sostener que llega a formar parte de la persona cuando esta no está en su rol investigador, sino en el metro, en el cine, o en la cola del banco. No es a través de un interruptor que actives ese tipo de mirada, considero que es algo que con el tiempo se va adquiriendo y en un momento pasas a ver toda la realidad a través de esa lente.

\subsubsection{Análisis}

Una vez que tenemos los datos recogidos en nuestros registros de observación, o resultantes de las entrevistas realizadas, así como algunos cuestionarios, o video grabaciones de fiestas referidas a ritos de paso por ejemplo, no tenemos el resultado de la investigación. Esta información necesita ser tratada para que al lector le llegue, como si fuese una película, ya montada, con la banda sonora incluida, y lista para proyectar. Así, esta información obtenida necesita de un montaje, y este montaje es el análisis del proceso etnográfico. Este análisis del material empírico necesita (en mayor o menor medida) de un marco teórico y de la capacidad analítica del investigador para interpretar los datos, para darles cierta coherencia y convertirlos en teoría.

Aclarar que no considero que necesariamente la cuestión analítica se encuentre al final del proceso investigador, de hecho, muy normalmente la capacidad de análisis del etnógrafo se intercala durante todo el proceso. Este análisis se realiza de un modo que expondré a continuación, siguiendo mi libro de metodología de cabecera, el de Hammersley y Atkinson. La teoría está desorganizada en todo el material del que el investigador dispone, y es tarea de este sacarla a la luz (igual que era tarea de Miguel Ángel desvelar la escultura que se encontraba dentro de la pieza de mármol de Carrara). Para ello, los autores proponen una serie de pasos. En un primer momento la lectura atenta y rigurosa del material recogido, para encontrar en él cuestiones significativas, relaciones entre ella, con el sentido común, o con la teoría previa. Se seleccionan, por lo tanto, las categorías analíticas sobresalientes del material: 
"El objetivo final, por supuesto, es alcanzar, una posición en la que se tenga un grupo estable de categorías y se pueda preparar una codificación sistemática de todos los datos en términos de esas categorías"34

Después se trabaja con la clase de categorías que resulten fundamentales para el análisis, y poder llevar a cabo relaciones con otros categorías. Anteriormente, y durante el proceso analítico el investigador cuenta con una o más teorías que fundamentan, esclarecen, cuestionan, refutan, etc., sus hipótesis de partida. Estas teorías por "triangulación" ayudan a depurar la investigación, e ir construyendo la teoría antropológica dependiente inductivamente de los datos empíricos obtenidos.

\subsection{Consideraciones sobre la cientificidad}

Anteriormente he hecho referencia a la cuestión de la antropología como ciencia. Intentaré dar una fundamentación mayor a este asunto. Uno de las cuestiones que se le pueden objetar a la antropología es su falta de objetividad, entendiendo la objetividad como "ausencia de interferencia". Siendo así el ideal de objetividad que el investigador no interviniese en la realidad modificándola, es decir, que se convirtiese en una mosca en la pared. Teniendo en cuenta la imposibilidad real de esta idea, lo único que para vencer esta limitación tiene el antropólogo en su mano, es su capacidad reflexiva, siendo consciente de que su rol investigador condiciona lo que está investigación. ¿Es este argumento suficiente para no conceder el carácter de ciencia a esta disciplina?. Si hiciéramos esto, según el principio de incertidumbre de Heisenberg también tendríamos que cuestionar la cientificidad de la física cuantifica:

"La presencia del observador modifica la naturaleza de lo que está observando. Bien sabemos que hundir un termómetro en un estanque de agua ¡modifica la temperatura que el termómetro mide supuestamente! [...] Podría en efecto responder que, mientras el agua del estanque está generalmente aquí antes de la llegada del observador, los discursos de los informantes, tal como se dan en las entrevistas, no preexisten a los del antropólogo. Esto es cierto y cualquier antropólogo bien formado es consciente de este problema, pero -

\footnotetext{
${ }^{34}$ Etnografia. Métodos de investigación o.c. p 231
} 
tengo ganas de decir- ¿y qué? -so what?-. De un lado, me cuesta imaginar una antropología que ipudiera hacerse sin antropólogo!’35

Como vemos, toda ciencia tiene sus sesgos, pero esto no invalida su condición científica. Lo que considero positivo de esta ciencia social es su humildad, en el sentido de que el antropólogo incluso en sus teorías tiene la precaución de delimitar el alcance de estas y su posible generalización o no. En algunos casos, las teorías a las que llega la antropología son teorías particulares de un lugar determinado o de un grupo social concreto (teorías de dimensión micro), pero esto no imposibilita su rigor científico, más allá de ello, creo que lo enaltece. Me gustaría terminar este apartado con las defensa de Candau:

"La Antropología es, pues, un sistema de conocimiento que posee un objeto determinado y un método propio. Esto es la definición misma de la ciencia, considerada como una empresa de conocimiento exacto y razonado de ciertos hechos particulares. En este sentido, la Antropología no difiere -por ejemplo- de la citología o de la genética, pues ellas también tienen un objeto y un método propios. Como éstas, es una ciencia, en este caso, la ciencia de la cultura" 36

\section{Relativismo lingüístico, cognitivo y cultural}

\subsection{Hipótesis de Sapir-Whorf}

Grosso modo, la hipótesis Sapir-Whorf viene a exponer una influencia o relación de los diversos lenguajes en el modo de cognición humana, y nuestras formas de ver el mundo.

Pero para entender mejor esta afirmación, es necesario que veamos las dos versiones de la hipótesis. Por un lado, tenemos la versión fuerte o determinista, que contiene en sí -implícita- la "tiranía del lenguaje". Esta versión del determinismo lingüístico, como ya se estará suponiendo, formula que el lenguaje determina la cognición humana. Esta versión ha sido criticada y desestimada por doquier. Según esta formulación, no percibimos la realidad tal y como se da en el exterior, sino a través de los mecanismos internos gramaticales de nuestra propia

\footnotetext{
${ }^{35}$ Candau, "La antropología, ciencia histórica y natural de la cultura" en: Revista de antropología social, n. 17 (2008), pp 278-279

${ }^{36}$ Ibid., p 276
} 
lenguaje. Según esta versión determinista, y echando mano también al primer Wittgenstein, y a su teoría figurativa del significado del Tractatus, y sin olvidar cierta influencia también de La filosofía del atomismo lógico de B. Russell, tenemos una necesaria isomorfía entre el lenguaje y el mundo. El modo en el que nos representemos la realidad será el que nos permita el lenguaje. Esto, siempre según, la primera formulación del determinismo lingüístico, y llevándola hasta sus últimas consecuencias, podríamos decir que lo que no se puede nombrar, no existe. $\mathrm{O}$ en términos wittgensteinianos "De lo que no se puede hablar hay que callar"37

Por otro lado, tenemos una versión más ampliamente aceptada que es la versión débil o del relativismo lingüístico. Esta afirma que existe una cierta influencia (que nunca es absoluta) del lenguaje en el pensamiento. Esta versión, menos controvertida, es sostenida por varios ejemplos etnográficos. Sobre todo ejemplos relativos al color, comos los estudios de F. Boas. Esta expresión de la hipótesis pone de manifiesto la esencia cultural de toda persona, el hombre como ser cultural, y la importancia del lenguaje en esa esencia humana, el lenguaje como cultura, o más correctamente, como parte fundamental de la cultura. Así, es claro comprobar una cierta influencia de nuestro lenguaje en nuestra forma de enfrentarnos al mundo ya que el hombre es un ser de "logos". Pero no determinando por él.

Antes de continuar, quería simplemente dejar constancia de que la hipótesis Sapir-Whorf pone de relieve la posible inconmensurabilidad de lenguajes y/o visiones del mundo debido a las diferentes gramáticas. Lo que implica también una variedad semántica, que dependiendo de la estructura gramatical de las lenguas se puede vencer con la traducción o no.

Esta hipótesis implica una relación entre la cultura, el lenguaje, y el comportamiento humano. Por un lado, y siguiendo la versión fuerte, no nos deja más opción que una cierta falta de libertad del individuo. Puesto que el hombre que se enfrenta al mundo, no lo hace con plena libertad, sino constreñido por una estructura gramatical determinada que le condiciona, ya de antemano, su realidad circundante y su cognición de la misma. Si nos fijamos en la otra versión, la del relativismo lingüístico, podemos decir algo similar, pero sin la carga despótica de antes. El hombre se enfrenta al mundo con una cultura y un

\footnotetext{
${ }^{37}$ Wittgenstein, Tractatus logico-philosophicus, Alianza, Madrid, 19997, p 7
} 
lenguaje, que por ello es un ser cultural, que le va a condicionar a la hora de enfrentarse al mundo, pero no le va a determinar. Por ello, de esta forma, el hombre se acerca al mundo con algo más libertad. Podemos observar como la cultura y la lengua son, en cierto modo, modos mediante los que el hombre se relaciona o conoce la realidad.

También se puede percibir una implicación acerca de la prioridad ontológica (no temporal) del lenguaje sobre el pensamiento humano en la versión determinista. Lo primero es el lenguaje, ya que sin él no podría darse el pensamiento, la cognición, porque pensamos con palabras, afirmación por otra parte más que criticada y desestimada. Esta radical relación lenguaje-pensamiento queda más distendida en la versión relativista, en la que como veremos a continuación, se pude pensar sin palabras, o lo que es lo mismo no es necesaria la causalidad de la palabra en los procesos cognitivos. Y para comprender mejor esto, vamos a pasar a ver que le objeta Candau a la hipótesis Sapir-Whorf.

Candau, en su artículo El lenguaje natural de los olores y la hipótesis Sapir-Whorf pone en cuestión la hipótesis a la que hemos estado, hasta ahora, dando vueltas. Y para ello, en lugar de centrase en los ejemplos de la percepción del color, tan estudiados etnográficamente, acude a otro tipo de percepción apenas atendida, la percepción del olfativa. Candau desestima la versión fuerte de la hipótesis, pero acepta, con cautela, la hipótesis relativista. Teniendo siempre en cuenta algo clave que demuestra en su artículo, la prioridad de la información sensorial sobre el lenguaje:

"[...] podemos sacar la conclusión de que, si bien no hay que excluir una influencia de la codificación verbal sobre la percepción olfativa, existen razones de peso para pensar que la elaboración de las informaciones sensoriales precede al lenguaje, si bien, a continuación, éste, actuando como un valioso auxiliar, permite comunicarlas y compartirlas" 38

La hipótesis de Sapir-Whorf se tambalea, cuando Candau pone de manifiesto, con su estudio, que en la percepción olfativa los olores primero se piensan y después ser formalizan verbalmente, con más o menos exactitud objetiva. Pero ofrece ciertas licencias al lenguaje ( $\sin$ excederse), por la influencia que el mismo tiene en nuestra manera de ver

\footnotetext{
${ }^{38}$ Candau, "El lenguaje natural de los olores y la hipótesis Sapir-Whorf” en: Revista de antropología social, n. 12 (2003), p 243
} 
el mundo, porque somos necesariamente seres culturales en los que el componente lingüístico está bastante presente.

Llegado este momento, podemos decir que la realidad es la misma, ante diversos ojos de diversas culturas, con sus respectivas diversas gramáticas. Pero que la forma de habérselas con la realidad depende, en cierto grado (aunque no totalmente), de la cada cultura, lengua y gramática utilizadas.

Un claro ejemplo ilustrativo lo encontramos en la investigación de Davidoff et al., acerca de la percepción y cognición del color entre sujetos ingleses y sujetos berinmo de Papúa-Nueva Guinea. Ante la misma realidad de pigmentos (la universalidad perceptiva del color), siendo la percepción la misma para todos los sujetos, la forma de nombrarlos difiere (relativismo lingüístico). Los sujetos ingleses reconocen ocho tipos diferentes de color, y los berinmo cinco (ver cuadro más abajo). Los autores comentan que hay una influencia lingüística considerable sobre la percepción de los colores:

"The differences between English and Berinmo allow a further critical test of the contrast between colour universals and linguistic relativity. Berinmo does not mark the distinction between blue and green, but it has a colour boundary (between 'nol' and 'wor') in a position that does not exist in English, 39

Con relación al relativismo lingüístico en materia de sensación, bien sea visual como acabamos de ver, u olfativa como la que trabaja el propio Candau, éste vendrá a sostener tres ideas: 1) no se sostiene la tesis del determinismo lingüístico; 2) se acepta mayormente la influencia lingüística relativa y; 3) esta última hipótesis no puede ser generalizable a todo dominio de la percepción.

Volviendo ahora al principio, al capítulo donde nos encontrábamos con Nietzsche y Wittgenstein, propongo que entendamos la construcción de la realidad a través de este prisma del relativo lingüístico.

${ }^{39}$ Davidoff-Davies-Roberson, «Colour categories in a stone-age tribe», Nature, 398 (1999), p 203 


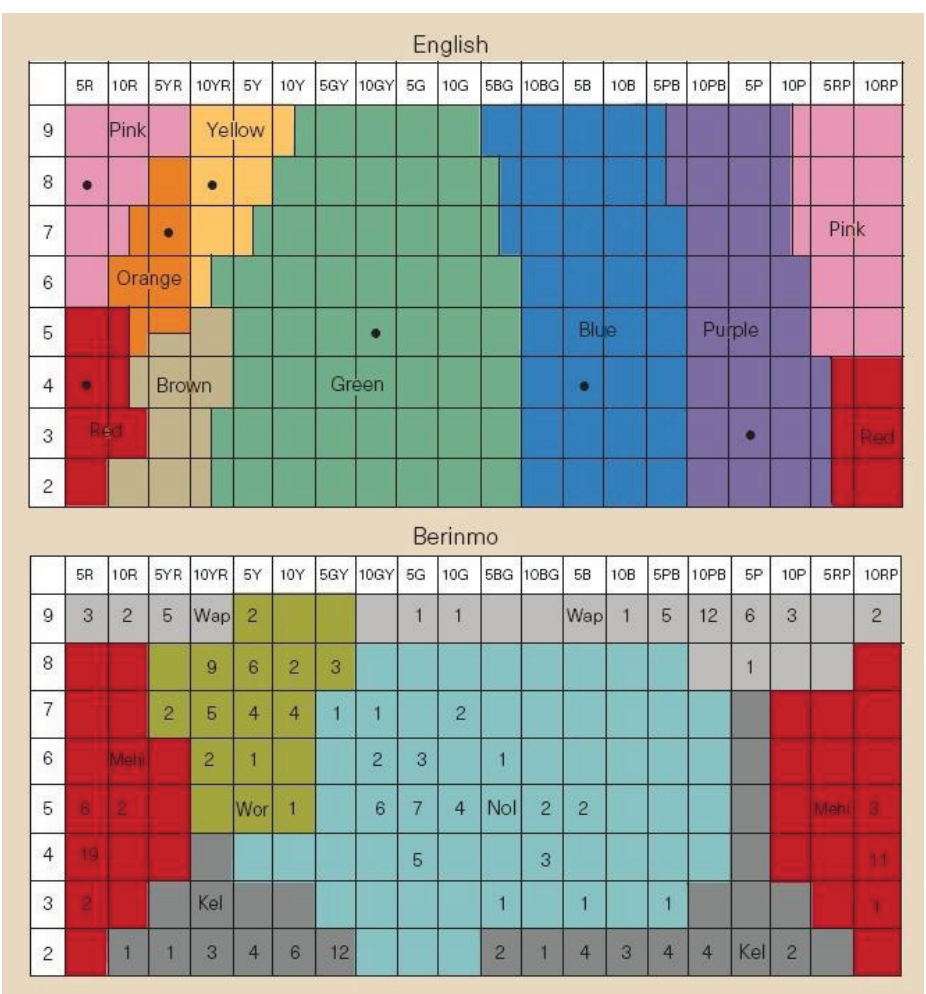

Figure 1: Distribution of English and Berinmo colour names. The Munsell system provides equally spaced samples in three dimensions, but is shown here as a Mercator projection of hue (horizontal axis) against lightness (vertical axis). The colours used to denote colour categories on these Mercator projections are for illustration only. Eight colour terms for English and five for Berinmo are shown. Dots on English naming data represent the position of focal colours. Numbers on the Berinmo naming data represent the number of subjects who designated that colour as best example of the category. R, red; Y, yellow; G, green; B, blue; P, pink ${ }^{40}$

${ }^{40}$ Gráfico del artículo de Davidoff et al., «Colour categories in a stone-age tribe», Nature, 398 (1999), pp. 203-204 


\subsection{El "pensamiento salvaje" de Lévi-Strauss}

Lo que Lévi-Strauss llama pensamiento salvaje consiste en un pensamiento mágico o mítico, que difiere (diré en un primer momento, más adelante iré puliendo esta idea) del pensamiento científico o moderno, característico del hombre civilizado. Este pensamiento salvaje no se mueve por la necesidad, por la utilidad, como normalmente se ha supuesto, sino que parte de un interés, de una curiosidad, como bien dice Lévi-Strauss corresponde a "exigencias intelectuales" 41 . Por tanto este pensamiento salvaje no es tan distinto de nuestro pensamiento "civilizado". Podemos advertir, como hace Lévi-Stauss que es un pensamiento con pretensión objetiva, igual que el científico.

Lévi-Strauss en el primer capítulo de El pensamiento salvaje, relaciona para su mejor comprensión, los conceptos "magia" y "ciencia". En lo que radicalmente se diferencian es que mientras la magia lleva implícito un determinismo casi absoluto, la ciencia actúa distinguiendo niveles. Sin embargo, pese a tal diferencia, Lévi-Strauss no ve una oposición entre magia y ciencia:

"en vez de oponer magia y ciencia, sería mejor colocarlas paralelamente, como dos modos de conocimiento, desiguales en cuanto a los resultados teóricos y prácticos [...], pero no por la clase de operaciones mentales que ambas suponen, y que difieren menos en cuanto a la naturaleza que en función de la clase de fenómenos a las que se aplican" 42

Como acabamos de ver, magia y ciencia, o pensamiento salvaje y pensamiento civilizado, no están tan lejanos uno de otro. Además, ambos tienen la misma finalidad que es poner en orden, clasificar, estructurar (como hace el bricoleur o el ingeniero) ese todo que es el universo al que se enfrentan. Pero ese modo de estructurar, es diverso, como Lévi-Strauss se adelanta al hablar del pensamiento primitivo así:

"es diferente porque su finalidad reside en alcanzar, por los medios más diminutos y económicos, una compresión general del universo [...]. Es decir, se trata de un modo de pensar que parte del principio de que si no se comprende todo no se puede explicar nada, lo cual es absolutamente contradictorio con la

\footnotetext{
${ }^{41}$ Leví-Strauss, pensamiento salvaje, FCE, Madrid, 2002, p 24

${ }^{42}$ Ibid, p 30
} 
manera de proceder del pensamiento científico, que consiste en avanzar etapa por etapa"43.

Lo menos arriesgado para cerrar este círculo abierto sobre el pensamiento salvaje creo que sería decir, que la mente humana es una y la misma para todos los seres humanos ("primitivos" o "civilizados"), y que lo que la hace a veces diferente es, no la forma de utilizarla (que más adelante veremos que es igual, pero iré adelantando dos términos clave: desinteresada e intelectual), sino el ámbito de hechos o acontecimientos a los que la aplicamos. Un claro ejemplo de Levi-Strauss de su artículo Pensamiento "primitivo" y mente "civilizada" ilustrará mejor mi exposición:

"Los pueblos ágrafos poseen un conocimiento extremadamente exacto de su medio y de todos sus recursos. Nosotros hemos perdido todas esas cosas, pero no ha sido en vano: ahora estamos en condiciones de guiar un automóvil sin correr el riesgo de ser aplastados en cualquier momento, y al final del día podemos arreglar la radio o el televisor,"44

En momentos históricos de la disciplina anteriores a LéviStrauss encontramos autores que también se preocuparon por la cuestión que aquí nos compete. Intentaré destacar los que, desde mi punto de vista, más pueden haber completar nuestro tema acerca de cuestiones sobre la mente.

Tylor reconoce en el primitivo a un ser inferior, incivilizado, cuyas capacidades cognoscitivas no están del todo desarrolladas. La actitud de éste, Evans-Pritchard ${ }^{45}$ la califica de intelectualista, y junto a ella designa de sociológica la postura que mantiene Lévy-Bruhl, ya que al tratar el modo de pensar como determinado por las representaciones colectivas, no reconoce las diferencias sobre los procesos de pensamiento individual. Aclarar que mientras que Tylor y Frazer insisten en las similitudes, Lévy-Bruhl resaltará las diferencias.

$$
\text { Lévy-Bruhl }^{46} \text { da una importancia principal a las }
$$
representaciones colectivas de la mentalidad primitiva. Estas representaciones colectivas, nos dirá Evans-Pritchard que son las ideas o

\footnotetext{
${ }^{43}$ LEVI-STRAUSS, C., Pensamiento "primitivo y mente "civilizada" en http://www.robertomarafioti.com/documentos/bibliografia $\% 20$ problematica $\% 20$ obligat oria/LEVISTRAUSS_1.doc

44 Ibid.

${ }^{45}$ En Las teorías de la religión primitiva

${ }^{46}$ En El alma primitiva
} 
creencia de una comunidad, de acuerdo con las cuales se juzga el mundo. Así, mientras que un hombre "primitivo" y otro "civilizado" perciban la misma cosa, cada uno, dependiendo de sus representaciones colectivas o creencias, lo pensará, y posteriormente lo clasificará de un modo distinto (algo muy similar a lo que vimos en el apartado anterior con referencia a la hipótesis Sapir-Whorf). Y algo parecido podemos decir de Durkheim, que prima las creencias del grupo social sobre el pensamiento individual. Esto es algo en lo que estos dos pensadores franceses van a diferir de los estudios ingleses.

Me gustaría poner en relación a Lévi-Strauss y a Lévy-Bruhl, para una mejor comprensión de sus propuestas. Así mientras Lévi-Strauss sostiene una igualdad de procesos mentales entre "primitivos" y "civilizados", Lévy-Bruhl mantiene la existencia de diferentes procesos mentales. Pero ambos pretenden defender al "primitivo" no considerándolo inferior (como hizo Tylor). Pero Lévy-Bruhl no lo conseguirá, y por ello categoriza el pensamiento de primitivo como prelógico (en el sentido de anterior a la lógica). Pero con todo ello, el primitivo de Lévy-Bruhl entiende un concepto tal como el de "imunu" que no es aprehensible para el hombre civilizado. Para Lévy-Bruhl serían las diferentes estructuras sociales las que expliquen las diferentes mentalidades. Lévi-Strauss no encuentra tal diferencia, y nos muestra como ambos son capaces de poner en estructura (que es una operación intelectual). Esta puesta en estructura la realizan tanto el bricoleur (o primitivo) y el ingeniero (o moderno). Ambos tienen un pensamiento lógico-intelectual, además de desinteresado.

Esta puesta en estructura de la que habla Lévi-Strauss es también reconocible en las clasificaciones primitivas que subraya Durkheim $^{47}$. Estas clasificaciones primitivas que son sistemas jerarquizados, Durkheim los destaca en todo tipo de sociedades y sostiene que no se distinguen mucho de las primeras clasificaciones científicas. Igualmente hemos visto como Lévi-Strauss pone de relieve que su puesta en estructura la realizan tanto primitivos como modernos. Y Durkheim, al igual que Lévi-Strauss ven estos sistemas clasificatorios una finalidad especulativa de agrupación de conocimiento. He de recalcar la naturaleza social que impregna el todo durkheimiano, que tiene mucho que ver como hemos visto con Lévy-Bruhl.

\footnotetext{
${ }^{47}$ En Clasificaciones primitivas (y otros ensayos de antropología positiva).
} 
Con relación a F. Boas ${ }^{48}$, creo que éste es un acertado antecedente del pensamiento lévi-straussiano. Boas se va a preguntar por la cuestión de las diferentes respuestas distintas ante un mismo hecho. Y para ello analizará las diferentes mentes (de diferentes razas). Para llegar a una conclusión parecida a la de Lévi-Strauss. Boas sostendrá concluyendo ya su artículo, que no hay diferentes mentes, es decir, que la estructura mental $\mathrm{y} / \mathrm{o}$ procesos mentales de las diferentes razas es la misma. Y que lo que hace que estas parezcan diferentes se debe (no a su psique) a los diferentes contenidos de la misma, así como también a sus diversos contextos sociales y/o geográficos.

Con lo visto hasta el momento, creo que podemos decir que en todo proceso cognitivo humano, la cultura que nos determina, tiene un papel fundamental en el momento de nuestra apertura cognoscitiva al mundo. Es tal la importancia de la cultura en el proceso de conocer, o anteriormente de percibir y catalogar, que no se pude realizar tal hecho sin ser un ser cultural. Con ello, lo que pretendo poner de manifiesto es la existencia de una unidad psíquica humana. Y que las posibles variaciones o diferencias, aparentes, que se puedan vincular de ella, no son propias de la mente, sino de las diferencias sociales o geográficas; en concreto, de las diferencias culturales y las tradiciones y creencias inmanentes a cada cultura.

\section{Conclusión (o posible punto de partida)}

Franz Boas fue el primero en acabar con la dicotomía entre pensamiento irracional o pre-lógico y pensamiento racional o civilizado. A través de un razonamiento erróneo como es el de creer que el hombre civilizado posee una mentalidad igualmente acorde y por detrimento, el hombre primitivo conlleva otro tipo inferior de mentalidad. Hemos llegado a considerar cómo superior nuestra intelectualidad, siendo está simplemente un producto de un determinado proceso histórico:

“muchas comunidades 'primitivas', lejos de ser subnacionales, pueden mostrar una mente funcionando en un plano más alto y complejo de racionalidad que el común de los hombres civilizados. No sabemos que civilización sea sinónimo de racionalidad. Puede que a estas tribus primitivas

${ }^{48} \mathrm{Y}$ a su artículo a La mente del hombre primitivo. The Journal of American Folklore, vol.14, n. 52 
sólo les haya faltado filósofos, cuya existencia, a su vez, puede depender de una prosperidad económica que sólo han alcanzado, durante el transcurso de la historia, unas pocas civilizaciones"

Si nunca nos hemos cuestionado la supuesta superioridad del europeo con respecto por ejemplo a grupos indígenas, dando por hecho que esta es así naturalmente, evolutivamente, etc., es normal, que lo anormal, lo que se aleja de lo normal (del europeo) sea considerado inferior. Pero esta mera de pensar, en simplemente el producto absurdo de la falta de reflexividad acerca de esta cuestión. Lo que hace la cultura al fin y al cabo es normalizar, homogeneizar, y todo lo que no está dentro de la supuesta normalidad es considerado no cultural (dentro de los patrones culturales de tal o cual grupo). Por lo tanto, este mismo pensamiento, si no nos detenemos en él nos llevará a conclusiones erróneas.

El otro día me comentaba alguien con increíble asombro: “¿tú sabes que los chinos se comen a los perros?", a lo que respondí: "sí claro, es cultural", y al rato comenté: "lo mismo que tú ahora mismo piensas de los chinos, piensan los musulmanes de ti cuando comes cerdo y los hindúes cuando comes ternera que es para ellos sagrada”. Esta anécdota en clave gastronómica cultural, me recuerda a otro ejemplo ilustrativo de Whorf:

"La lengua hopi posee un nombre que abarca todo clase o ser que vuela, con la excepción de los pájaros, cuya clase viene indicada por otro nombre. Se puede decir que el nombre formal indica la clase (CV-P) clase de vuelo menos pájaro. De esto modo el hopi llama insecto, avión o aviador mediante la misma palabra, y no siente ninguna dificultad en hacerlo así. [...] Esta clase nos parece bastante grande e inclusiva, pero lo mismo le parecería al esquimal nuestra clase de 'nieve'. Utilizamos la misma palabra para la nieve que cae, la nieve que está en el suelo, y la nieve endurecida como hielo, cualquiera que sea su situación. Para un esquimal seria inconcebible esta palabra que lo incluye todo; él diría que la nieve que cae, la nieve que está en el suelo, etc., son algo diferente desde el punto de vista sensitivo y operacional"50

Ahora bien, ¿quién se atrevería a decir que es más verdad nuestra conocimiento univoco de la nieve o la polivocidad del esquimal?, ¿quién a sostener que el chino es un despiadado por comer perro?, cerrando el círculo que abrí al principio, ¿quién se atrevería a sostener

${ }^{49}$ Lenguaje, pensamiento y realidad o.c. p 98

${ }^{50}$ Ibid., 244 
que una forma de jugar al ferrocarril el más válida que la otra?, ¿quién a decir que el esquimal o el berinmo no conocen con precisión los colores?. Tanto un ejemplo como otros, nos demuestran que los diversos aspectos, ya sean gastronómicos, cognoscitivos, lingüísticos, etc., no están por encima de los sistemas de culturales, sino que deben ser analizados desde dentro de los mismos, comprendidos, explicados, desde cada una de las grupos sociales culturales donde se enmarcan.

Como hemos visto a través de ejemplos culturales, las mismas categorías que nosotros conocemos no son traducibles literalmente a otras culturas, o lo que es lo mismo, la inconmesurabilidad cultural hace que no compartamos los mismos esquemas cognoscitivos (como también hemos visto en Lévi-Strauss por ejemplo, no debido a una etapa de desarrollo mental determinada, sino a una dimensión cultural distinta con la que ha de habérselas toda cultura), y que determinadas intuiciones como las del "espacio" y "tiempo" que determinan gran parte de nuestra manera de comportarnos, conocer y ser, no puedan ser universalizables, sino que respondan a unos determinados esquemas de pensamiento y a una historia concreta.

Toda forma de enfrentarnos a la realidad es inconsciente (la cultura es inconsciente y todos somos seres culturales), en el sentido en que no nos planteamos que pueda haber otra forma, debido a que históricamente, se nos ha enseñado esta (y no la otra). El primer paso, es acabar con este pensamiento etnocentrista, y considerar la posibilidad de que "el otro" haga las cosas de otra manera y sin embargo, no estén mejor o peor, simplemente que sean diferentes, y tan válidas como las nuestras. Y esta diferencia conlleva una lógica coherente dentro de su estructura cultural, lógica que a simple vista no advertimos, por la sencilla razón de que no compartimos sus mismos códigos culturales, pero ello no quiere decir que no exista:

"A través del lenguaje, la civilización occidental ha hecho un análisis provisional de la realidad, y mantiene como definitivo este análisis, sin aceptar correcciones. Las únicas correcciones a introducir deben ser las de todas aquellas otras lenguas que, como consecuencia de una evolución independiente, han llegado a análisis diferentes, pero no por ello menos lógicos y provisionales" "(la cursiva en mía).

\footnotetext{
${ }^{51}$ Ibid., p 275
} 
Vanesa Blanco Gallardo es licenciada en Filosofía por la Universidad de Málaga, y licenciada en Antropología social y cultural por la Universidad Complutense de Madrid. Sus intereses como investigadora están relacionados con el ámbito de la antropología de cuerpo, movimientos contraculturales, modificación corporal. Actualmente está cursando el doctorado en la rama de Antropología, investigando para su DEA cuestiones sobre políticas públicas en inmigración en la Comunidad de Madrid

Este trabajo, Más allá de occidente. Lo que la antropología puede enseñar a la filosofía, reproduce la ponencia de Vanesa Blanco del 6 de abril de 2010, en el centro cultural Vicente Aleixandre, Alhaurín de la Torre (Málaga), y que supuso el acto final con el que se cerró el ciclo de seminarios en torno a la verdad organizados por "Claridades. Revista de filosofía" en colaboración con el área de filosofía del departamento de filosofía de la Universidad de Málaga. 